\title{
Continuous surveillance of points by rotating floodlights
}

\author{
S. Bereg*1, J. M. Díaz-Báñez ${ }^{\dagger 2}$, M. Fort ${ }^{\ddagger 3}$, M. A. Lopez ${ }^{\S 4}$, P. Pérez-Lantero ${ }^{\llbracket 5}$, and J. Urrutia ${ }^{\| 6}$ \\ ${ }^{1}$ Department of Computer Science, University of Texas at Dallas, USA. \\ ${ }^{2}$ Departamento Matemática Aplicada II, Universidad de Sevilla, Spain. \\ ${ }^{3}$ Departament d'Informàtica i Matemàtica Aplicada, Universitat de Girona, Spain. \\ ${ }^{4}$ Department of Computer Science, University of Denver, USA. \\ ${ }^{5}$ Escuela de Ingeniería Civil en Informática, Universidad de Valparaíso, Chile. \\ ${ }^{6}$ Instituto de Matemáticas, UNAM, Mexico.
}

\begin{abstract}
Let $P$ and $F$ be sets of $n \geq 2$ and $m \geq 2$ points in the plane, respectively, so that $P \cup F$ is in general position. We study the problem of finding the minimum angle $\alpha \in[2 \pi / m, 2 \pi]$ such that one can install at each point of $F$ a stationary rotating floodlight with illumination angle $\alpha$, initially oriented in a suitable direction, in such a way that, at all times, every target point of $P$ is illuminated by at least one light. All floodlights rotate at unit speed and clockwise. We give an upper bound for the 1-dimensional problem and present results for some instances of the general problem. Specifically, we solve the problem for the case in which we have two floodlights and many points, and give an upper bound for the case in which there are many floodlights and only two target points.
\end{abstract}

\section{Introduction}

Illumination problems are well known in Discrete and Computational Geometry [4]. Let $P$ be a set of $n \geq 2$ targets and $F$ a set of $m \geq 2$ floodlights, both defined in the plane. We assume that $P \cup F$ is in general position and that all floodlights rotate clockwise at unit speed. We say that $F$ covers $P$ with illumination angle $\alpha \geq 2 \pi / m$ if there is a suitable initial orientation of each light so that, at all times, each target point of $P$ is illuminated by at least one floodlight. We

\footnotetext{
*Email: besp@utdallas.edu. Partially supported by project MEC MTM2009-08652.

†Email: dbanez@us.es. Partially supported by project MEC MTM2009-08652 and ESF EUROCORES programme EuroGIGA-ComPoSe IP04-MICINN Project EUI-EURC-20114306.

†Email: mfort@ima.udg.edu. Partially supported by the Spanish MCI grant TIN2010-20590-C02-02.

Email: mlopez@du.edu.

IEmail: pablo.perez@uv.cl. Partially supported by project CONICYT, FONDECYT/Iniciación 11110069 (Chile), and project MEC MTM2009-08652.

"Email: urrutia@matem.unam.mx. Partially supported by project MEC MTM2009-08652.
}

consider the problem of finding the minimum angle $\alpha=\alpha(P, F) \in[2 \pi / m, 2 \pi]$ and initial orientation of each floodlight so that $F$ covers $P$.

Our general problem can be considered a discretization of the one studied by Kranakis et al. [2]. Given the locations of $m$ floodlights (i.e. antennae) and a region, their problem asks to schedule the lights so that the entire region is covered at all times. The scheduling of static floodlights for covering a given region was first considered by Bose et al. [1. Research related to our problem can be found in a number of domains, including art gallery and related problems, multi-target tracking, and multi-robot surveillance tasks [3, 4]. A complete review of these fields can be found in [4].

We present results for some cases of our general problem: the elements of $P \cup F$ are located on a given line (Section 2), the two-dimensional version with two floodlights (Section 3), and the problem in the plane with two target points (Section 4).

Given points $u, v$ in the plane, $\ell(u, v)$ denotes the line containing both $u$ and $v$. We identify any floodlight by the point where it is installed. For any floodlight $f$, at any instance of time, the region illuminated by $f$ is delimited by rays $f^{-}$and $f^{+}$, starting at $f^{-}$ and ending at $f^{+}$in the clockwise direction. We say that we configure $f$ with angle $\beta$ if the angle between $f^{+}$and the positive $x$-axis is equal to $\beta$. Given that all floodlights rotate at the same speed, it suffices to consider only the interval of time $[0,2 \pi)$.

\section{Points and floodlights on a line}

We first consider the case in which the points of $P$ and the floodlights of $F$ lie on a line $\mathcal{L}$, say the $x$ axis. Kranakis et al. 22] considered the case in which the floodlights are located on a line. They showed that the entire line can be illuminated by $m$ rotating floodlights using illumination angle $3 \pi / m$ and this bound is tight. This can be viewed as a special case of our problem where $n \geq m+1$ and each of the $m+1$ segments determined by $F$ contains at least one point of 
$P$. We consider other cases and show that the illumination angle is smaller than $3 \pi / m$ for some of them.

Partition $P$ into $k-1(k \geq 2)$ maximal intervals $s_{1}, s_{2}, \ldots, s_{k-1}$, from left to right, each of which contains elements of $P$ but no elements of $F$. Let $F_{1}$ denote the elements of $F$ to the left of $s_{1}, F_{i}$ $(i=2, \ldots, k-1)$, the elements of $F$ between $s_{i-1}$ and $s_{i}$, and $F_{k}$, the elements of $F$ to the right of $s_{k-1}$. Let $m_{i}=\left|F_{i}\right|$ for $i=1, \ldots, k$. Observe that $m_{1}, m_{k} \geq 0$, $m_{i} \geq 1$ for $i=2, \ldots k-1$, and $m_{1}+m_{2}+\ldots+m_{k}=m$.

Lemma 1 Two floodlights $f_{1}$ and $f_{2}$ with illumination angle $\alpha$, belonging to the same set among $F_{1} \cup$ $F_{k}, F_{2}, F_{3}, \ldots, F_{k-1}$, can be configured to cover $P$ during $2 \alpha$ time in total. Furthermore, if two floodlights of $F$ cover $P$ with angle $\alpha<3 \pi / 2$, then they must belong to a same set among $F_{1} \cup F_{k}, F_{2}, F_{3}, \ldots, F_{k-1}$.

Proof. Suppose that both $f_{1}$ and $f_{2}$ belong to a set $F_{i}(i=1, \ldots, k)$, and assume w.l.o.g. that $f_{1}$ is to the left of $f_{2}$. Configure $f_{1}$ with angle zero and $f_{2}$ with angle $\pi$ (see top of Figure 1a). At time $t=\pi$ the configuration of $f_{1}$ and $f_{2}$ is as shown in the bottom of Figure 1a. Since there is no element of $P$ in the segment connecting $f_{1}$ and $f_{2}$ then all elements of $P$ are illuminated during intervals $[0, \alpha]$ and $[\pi, \pi+\alpha]$, $2 \alpha$ time in total.

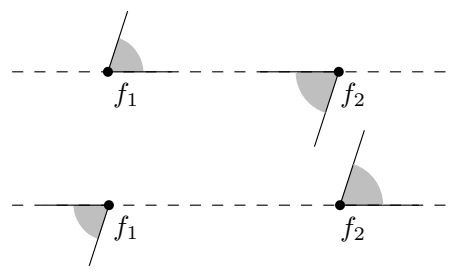

(a)

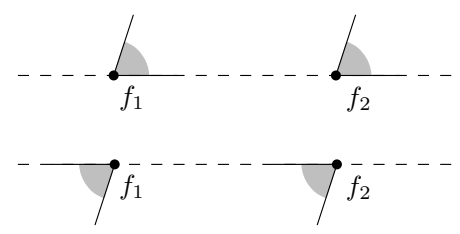

(b)

Figure 1: Proof of Lemma 1 (a) Case where $f_{1}$ and $f_{2}$ belong a same set $F_{i}$. (b) Case where $f_{1} \in F_{1}$ and $f_{2} \in F_{k}$.

Suppose now that $f_{1} \in F_{1}$ and $f_{2} \in F_{k}$. Configure both $f_{1}$ and $f_{2}$ with angle zero (see top of Figure 1b). At time $t=\pi$ the configuration of $f_{1}$ and $f_{2}$ is as shown in the bottom of Figure 1b. Since all elements of $P$ belong to the segment connecting $f_{1}$ and $f_{2}$ then all elements of $P$ are illuminated during intervals $[0, \alpha]$ and $[\pi, \pi+\alpha], 2 \alpha$ time in total.

For the second part of the lemma, let $f$ and $g$ be the two lights that cover $P$. Clearly $\alpha \geq \pi$. Assume, w.l.o.g., that $g \notin F_{1} \cup F_{k}$ and that $f$ is to the left of g. Also, let $Q \subseteq P$ denote the set of targets to the right of $f$. Light $f$ alone can cover $Q$ for an interval of length $\alpha$, while $g$ alone can cover $Q$ for $\alpha-\pi$ only. Since $\alpha<3 \pi / 2$, an interval of length $2 \pi-\alpha>\pi / 2$ is unaccounted for by $f$, but $g$ can pick up at most $\alpha-\pi<\pi / 2$ of this. Hence, $f$ and $g$ must belong to the same set. The claim does not hold if $\alpha \geq 3 \pi / 2$.

Lemma 2 Three floodlights $f_{1}, f_{2}$, and $f_{3}$ with illumination angle $\alpha<\pi$, can be configured so that, together, they cover the whole line $\mathcal{L}$ (hence, $P$ ) during $2 \alpha$ time in total.

Proof. The proof can be obtained from [2]. Assume, w.l.o.g., that $f_{1}, f_{2}$, and $f_{3}$ appear in this order from left to right. Configure $f_{1}, f_{2}$, and $f_{3}$ with angle zero, $\pi$, and zero, respectively (top of Figure 2). At time $t=\pi$ the configuration is as shown at the bottom of Figure 2, During interval $[0, \alpha]$ the line is illuminated by $f_{1}$ and $f_{2}$, and during interval $[\pi, \pi+\alpha]$, by $f_{2}$ and $f_{3}$. The result follows.

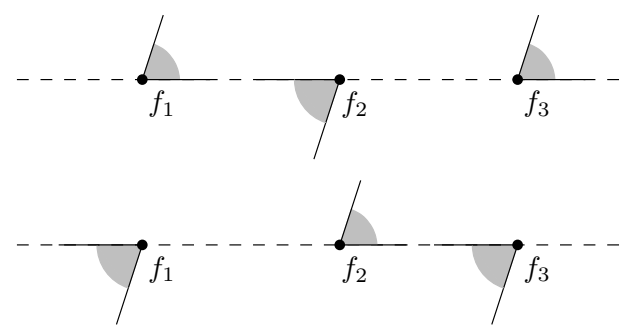

Figure 2: Proof of Lemma 2

Theorem 3 If all floodlights belong to the same set among $F_{1} \cup F_{k}, F_{2}, F_{3}, \ldots, F_{k-1}$, then $\alpha(P, F)=$ $2 \pi / m$, which is optimal. Otherwise, $\alpha(P, F)$ satisfies:

$$
\alpha(P, F) \leq \min \left\{\frac{3 \pi}{m}, \frac{2 \pi}{m-Q+2\left\lfloor\frac{Q}{3}\right\rfloor}\right\}
$$

where $Q$ denotes the number of odd numbers in the set $\left\{m_{1}+m_{k}, m_{2}, \ldots, m_{k-1}\right\}$.

Proof. Obviously $\alpha(P, F) \geq 2 \pi / m$ in all cases. All lights belong to the same set iff $k=2$, or $k=3$ and $m_{1}=m_{3}=0$. In both cases, illumination angle $\alpha=2 \pi / m$ is sufficient. Assume first that $k=2$ and let $F_{1}=\left\{f_{1}, \ldots, f_{m_{1}}\right\}$ and $F_{2}=\left\{f_{1}^{\prime}, \ldots, f_{m_{2}}^{\prime}\right\}$. Floodlight $f_{i}$ is configured with angle $(i-1) \alpha$ for $i=1, \ldots, m_{1}$, and floodlight $f_{j}^{\prime}$, with angle $\pi-j \alpha$ for $j=1, \ldots, m_{2}$ (see Figure 3). Then, at any time $t \in\left[0, m_{1} \alpha\right) P$ is covered by a member of $F_{1}$ and, at any time $t \in\left[m_{1} \alpha, 2 \pi\right)$, by a member of $F_{2}$. Assume now that $k=3$ and $m_{1}=m_{3}=0$. Then, $F=F_{2}=\left\{f_{1}, \ldots, f_{m}\right\}$. By configuring $f_{i}$ with angle $2 i \pi / m, P$ is covered by $F$. This proves the optimal result when all lights belong to the same set. 


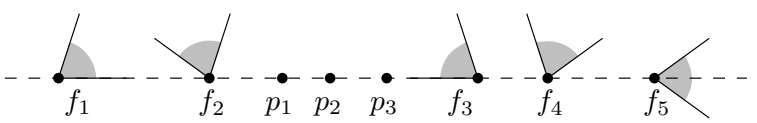

Figure 3: Two groups of floodlights $F_{1}=\left\{f_{1}, f_{2}\right\}$ and $F_{2}=\left\{f_{1}^{\prime}, f_{2}^{\prime}, f_{3}^{\prime}\right\}=\left\{f_{3}, f_{4}, f_{5}\right\}$, where $m_{1}=2$ and $m_{2}=$ 3 , and the configuration with angle $\alpha=2 \pi / 5$.

If neither of the two cases above occurs, we configure the floodlights to satisfy inequality (1) as follows. We consider the case $m_{1}=m_{k}=0$ and $m_{i}=1$ $(i=2, \ldots, k-1)$ separately because the result follows immediately from [2], since our problem is equivalent to illuminating the whole $x$-axis. In this case $m=Q$ and $\alpha(P, F)=3 \pi / m=\min \left\{\frac{3 \pi}{m}, \frac{2 \pi}{m-Q+2\left\lfloor\frac{Q}{3}\right\rfloor}\right\}$ which is also optimal.

For the remaining cases, we can only obtain an upper bound on the optimal illumination angle. We proceed as follows. Pair the elements of $F$ into $N=\left\lfloor\frac{m_{1}+m_{k}}{2}\right\rfloor+\left\lfloor\frac{m_{2}}{2}\right\rfloor+\ldots+\left\lfloor\frac{m_{k-1}}{2}\right\rfloor=$ $\frac{m-Q}{2}$ pairs $\left(f_{1,1}, f_{1,2}\right), \quad\left(f_{2,1}, f_{2,2}\right), \ldots,\left(f_{N, 1}, f_{N, 2}\right)$ so that the elements of each pair belong to a same set among $F_{1} \cup F_{k}, F_{2}, F_{3}, \ldots, F_{k-1}$. Group the remaining $Q$ floodlights into $M=\left\lfloor\frac{Q}{3}\right\rfloor$ triples $\left(f_{1,1}^{\prime}, f_{1,2}^{\prime}, f_{1,3}^{\prime}\right), \ldots,\left(f_{M, 1}^{\prime}, f_{M, 2}^{\prime}, f_{M, 3}^{\prime}\right)$ (leaving at most two ungrouped). Let $\alpha=\frac{2 \pi}{m-Q+2\left\lfloor\frac{Q}{3}\right\rfloor}=$ $\frac{2 \pi}{2 N+2 M}$. We now schedule the floodlights as follows. Configure $f_{i, 1}$ and $f_{i, 2}$ with angles $(i-1) \alpha$ and $\pi+(i-1) \alpha$, respectively, for $i=1, \ldots, N$; and configure $f_{j, 1}^{\prime}, f_{j, 2}^{\prime}$, and $f_{j, 3}^{\prime}$ with angles $(N+j-1) \alpha$, $(N+j-1) \alpha$, and $\pi+(N+j-1) \alpha$, respectively, for $j=1, \ldots, M$. Finally, arbitrarily configure the remaining floodlights (at most two). The correctness of this configuration follows from lemmas 1 and 2

\section{Many points and two lights}

In this section we consider the case of two floodlights $f_{1}$ and $f_{2}$, i.e., $m=2$. Let $p_{1}, \ldots, p_{n}$ denote the elements of $P$. Assume w.l.o.g. that line $\ell\left(f_{1}, f_{2}\right)$ is horizontal and that $f_{1}$ is located to the left of $f_{2}$. Given any target point $p_{i}(i=1, \ldots, n)$, let $\theta_{i} \in[0, \pi)$ denote the angle at $p_{i}$ in the triangle $\triangle p_{i} f_{1} f_{2}$. If there are points from $P$ on both sides of the line $\ell\left(f_{1}, f_{2}\right)$, then we define two angles $\beta^{+}$and $\beta^{-}$as the maximum of $\theta_{i}$ over all points $p_{i}$ above and below $\ell\left(f_{1}, f_{2}\right)$, respectively (see Figure 4a). Otherwise, all the points of $P$ are on the same side of $\ell\left(f_{1}, f_{2}\right)$ and we define two angles, $\beta_{\max }$ and $\beta_{\text {min }}$, as the largest and smallest $\theta_{i}$ over all points $p_{i}$, respectively (see Figure $4 \mathrm{~b}$.

Theorem 4 (Two floodlights) For $m=2, n \geq 2$ : (1) If there are points of $P$ on both sides of $\ell\left(f_{1}, f_{2}\right)$ then $\alpha(P, F)=\pi+\frac{\beta^{+}+\beta^{-}}{2}$.

(2) If all the points of $P$ lie on one side of $\ell\left(f_{1}, f_{2}\right)$ then $\alpha(P, F)=\pi+\frac{\beta_{\max }-\beta_{\min }}{2}$.

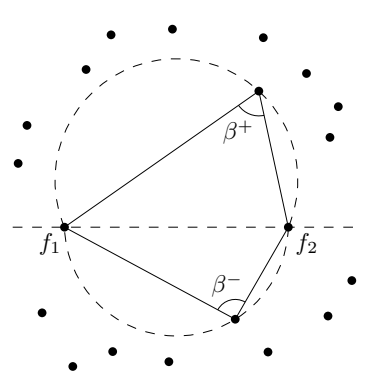

(a)

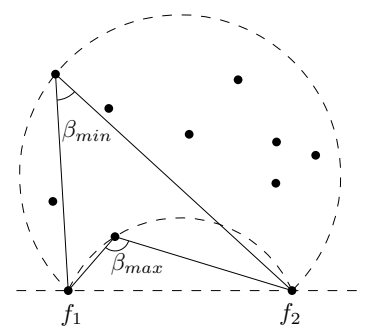

(b)
Figure 4: (a) $\beta^{+}$and $\beta^{-}$. (b) $\beta_{\max }$ and $\beta_{\min }$.

Proof. First, we prove part (1) of the theorem. We configure floodlights $f_{1}$ and $f_{2}$ initially as follows. Let $A f_{1} B f_{2}$ be the quadrilateral such that angle $\angle f_{1} A f_{2}$ is equal to $\beta^{+}$, angle $\angle f_{2} B f_{1}$ is equal to $\beta^{-}$, and points $f_{1}$ and $f_{2}$ are symmetric with respect to the line $\ell(A, B)$ as shown in Figure 5 a.

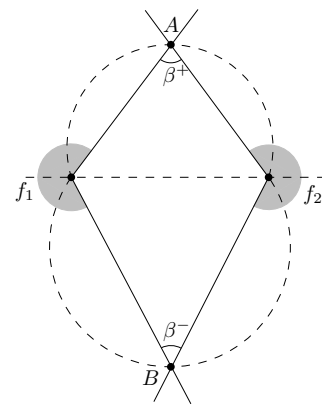

(a)

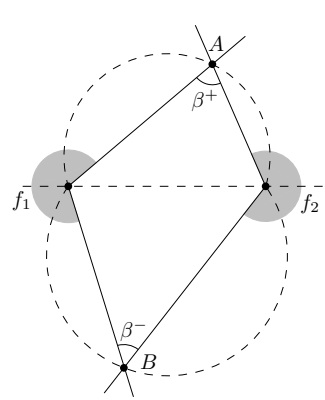

(b)

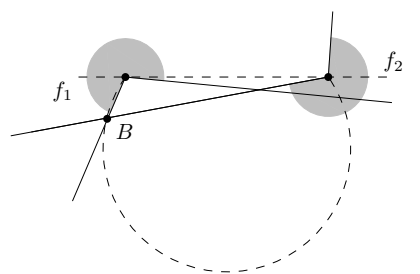

(c)

Figure 5: (a) Initial position. (b) (c) General position.

Since $f_{1}$ and $f_{2}$ rotate at unit speed, it always holds that $\angle f_{1} A f_{2}=\beta^{+}$and $\angle f_{2} B f_{1}=\beta^{-}$(see Figure $5 \mathrm{~b}$ ). Furthermore, the region not illuminated by the lights is always a subset of the interior of the union of the triangles $\triangle f_{1} A f_{2}$ and $\triangle f_{2} B f_{1}$ (see Figures $5 \mathrm{~b}$ and $5 \mathrm{c}$ ), and it never contains points of $P$ by the definition of $\beta^{+}$and $\beta^{-}$. It remains to show that any illumination angle smaller than $\pi+\frac{\beta^{+}+\beta^{-}}{2}$ is not feasible.

Suppose that, initially, floodlight $f_{i}, i=1,2$ covers angles in the interval $\left[\alpha_{i}, \beta_{i}\right]$. First we show that these intervals cover all possible directions in $[0,2 \pi]$. If, to the contrary, a direction $t$ is not covered by $\left[\alpha_{1}, \beta_{1}\right] \cup$ $\left[\alpha_{2}, \beta_{2}\right]$, then there is a rotation such that $p_{i}$ is not illuminated, where $p_{i}$ is the point above the $x$-axis and $\angle f_{2} p_{i} f_{1}=\beta^{+}$, a contradiction. Therefore, the 
floodlight intervals overlap as shown in Figure 6a.

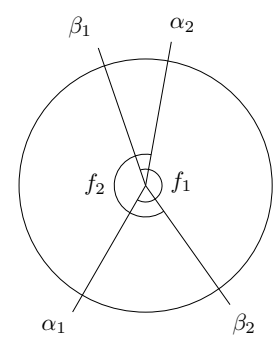

(a)

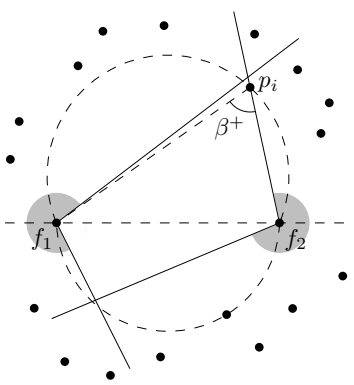

(b)
Figure 6: The floodlight intervals.

We show now that the overlapping interval $\left[\alpha_{2}, \beta_{1}\right]$ has length at least $\beta^{+}$. Suppose to the contrary that it is smaller than $\beta^{+}$. Consider the rotation by the angle $\gamma$ such that the $\beta_{2}$-ray of floodlight $f_{2}$ passes through the point $p_{i}$ defining $\beta^{+}$(see Figure 6b). Then the $\alpha_{1}$-ray of floodlight $f_{1}$ will not cover $p_{i}$ since the angle between the two rays is less than $\beta^{+}$. Therefore $p_{i}$ is not covered if the rotation angle is slightly smaller than $\gamma$, a contradiction. Similarly, the overlapping interval $\left[\alpha_{1}, \beta_{2}\right]$ has length at least $\beta^{-}$. If the illumination angle is $\alpha$ then $2 \alpha \geq 2 \pi+\beta^{+}+\beta_{i}$. The claim of part (1) follows.

To prove part (2) of the theorem we configure floodlights $f_{1}$ and $f_{2}$ at the beginning as follows. Let $A f_{1} B f_{2}$ be a quadrilateral such that $\angle f_{1} A f_{2}=\beta_{\text {min }}$ and $\angle f_{2} B f_{1}=\beta_{\max }$ and points $f_{1}$ and $f_{2}$ are symmetric about line $\ell(A, B)$ as shown in Figure $7 \mathrm{a}$. The argument is similar to the proof of part (1) since the points $A$ and $B$ move along arcs shown in Figure $7 \mathrm{a}$. The uncovered part is either a region below $C f_{1} B f_{2} D$, shown in Figure $7 \mathrm{a}$, or the wedge $X Y Z$, shown in Figure $7 \mathrm{c}$. In any case the area between the two arcs defined by $\beta_{\min }$ and $\beta_{\max }$ is always illuminated.

The optimality of angle $\pi+\frac{\beta_{\max }-\beta_{\min }}{2}$ can be shown similarly to the proof of part (1).

\section{Many lights and two points}

Let $p_{1}$ and $p_{2}$ denote the elements of $P$ and $f_{1}, \ldots, f_{m}$ denote the elements of $F$. Let $\theta_{i}(i=1, \ldots, m)$ denote the angle by which line $\ell\left(f_{i}, p_{1}\right)$ has to be rotated clockwise with center $f_{i}$ to become line $\ell\left(f_{i}, p_{2}\right)$. Assume w.l.o.g. that $\theta_{1} \leq \theta_{2} \leq \ldots \leq \theta_{m}$.

Lemma 5 If $n=2$ then $\alpha(P, F) \leq \frac{2 \pi}{m}+\frac{\theta_{m}-\theta_{1}}{m}$.

Proof. It suffices to prove that for $\alpha=\frac{2 \pi}{m}+\frac{\theta_{m}-\theta_{1}}{m}$ the $m$ floodlights can be configured properly. Configure $f_{1}$ arbitrarily and, for $i=1, \ldots, m-1$, configure $f_{i+1}$ to start illuminating point $p_{2}$ at the time $f_{i}$ stops illuminating it. Since $\alpha \geq 2 \pi / m$ then $p_{2}$ is

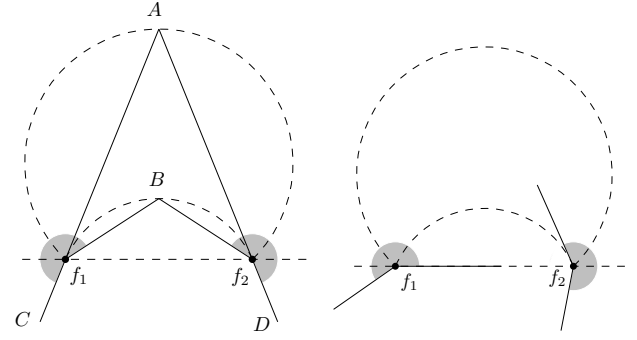

(a)

(b)

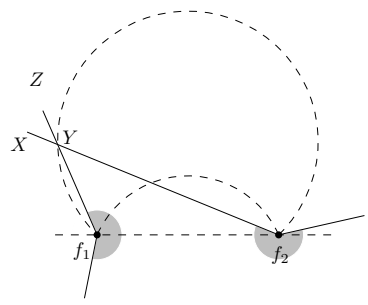

(c)

Figure 7: The floodlight intervals.

always illuminated. Observe that $p_{1}$ is illuminated at some time by both $f_{i}$ and $f_{i+1}$ since $\theta_{i} \leq \theta_{i+1}$. Then the time spam in which $p_{1}$ is illuminated by $f_{i}$ and not by $f_{i+1}$ is equal to $\alpha+\theta_{i}-\theta_{i+1}$. Since $\sum_{i=1}^{m-1}\left(\alpha+\theta_{i}-\theta_{i+1}\right)+\alpha=m \alpha+\theta_{1}-\theta_{m}=2 \pi$ then $p_{1}$ is always illuminated.

One can build examples, as the following one, in which $\theta_{1}<\theta_{m}$ and $\alpha$ is the theoretical minimum (i.e. $\alpha=$ $2 \pi / m)$, showing that $\alpha=\frac{2 \pi}{m}+\frac{\theta_{m}-\theta_{1}}{m}$ is not always optimal. Let $m=8$ and $\left(\theta_{1}, \theta_{2}, \theta_{3}, \theta_{4}, \theta_{5}, \theta_{6}, \theta_{7}, \theta_{8}\right)=$ $\left(\frac{\pi}{4}, \frac{\pi}{4}, \frac{\pi}{2}, \frac{\pi}{2}, \frac{\pi}{2}, \frac{\pi}{2}, \frac{3 \pi}{4}, \frac{3 \pi}{4}\right)$. Then $f_{1}, \ldots, f_{8}$ can be configured with $\alpha=2 \pi / m$ so that $p_{1}$ is illuminated in the (circular) order $f_{1}, f_{3}, f_{8}, f_{2}, f_{4}, f_{5}, f_{6}, f_{7}$ and $p_{2}$ in the order $f_{6}, f_{1}, f_{7}, f_{3}, f_{2}, f_{8}, f_{4}, f_{5}$. Thus, it becomes interesting to decide whether $\alpha(P, F)=2 \pi / m$ when $\theta_{1}, \ldots, \theta_{m}$ are all multiples of $2 \pi / m$.

Acknowledgements. The problem studied here was introduced and partially solved during the VI Spanish Workshop on Geometric Optimization, June 2012, El Rocío, Huelva, Spain. The authors would like to thank other participants for helpful comments.

\section{References}

[1] J. Bose, L. Guibas, A. Lubiw, M. Overmars, D. Souvaine, and J. Urrutia. The floodlight illumination problem. Int. J. Comp. Geom., 7:153-163, 1997.

[2] E. Kranakis, F. MacQuarie, O. Morales, and J. Urrutia. Uninterrupted coverage of a planar region with rotating directional antennae. In $A D H O C-N O W$, pages 56-68, 2012.

[3] K. Sugihara, I. Suzuki, and M. Yamashita. The searchlight scheduling problem. SIAM J. Comput., 19(6):1024-1040, 1990.

[4] J. Urrutia. Art gallery and illumination problems. In Hdbk. of Comp. Geom., pages 973-1027, 2000. 